\title{
KRAS NM_004985.3:c.35_36delGTinsAA
}

National Cancer Institute

\section{Source}

National Cancer Institute. KRAS NM 004985.3:C.35 36delGTinsAA. NCI Thesaurus. Code C98374.

A complex substitution where the nucleotide sequence at positions 35 through 36 of the coding sequence of the KRAS gene has changed from guanine-thymine to adenineadenine. 